Linda J. Fisher, U.S. Environmental Protection Agency, letter to Henry A. Waxman, U.S. Congress. May 1990, Attachment 1. Food Use Pesticides which have been Evaluated for Carcinogenicity.

The authors wish to thank Jennifer Boursier, Legislative Coordinator for the California Department of Food and Agriculture, and Jerry J. Baron,
Associate Coordinator for IR-4 National Headquarters, Rutgers University, for their assistance in this project.

Michael W. Stimmann is Statewide Pesticide Coordinator, UC DANR, based at UC Davis; and Mary P. Ferguson is Special Assistant for Microbial Pesticide Registration for IR-4, a nationwide program to support the registration of pesticides on minor crops, and is also based at UC Davis.

\title{
Alternatives to targeted pesticides: the DANR database
}

\author{
Frank G. Zalom - Joyce F. Strand
}

Late in 1989, the Division of Agriculture and Natural Resources (DANR) initiated an inventory of alternatives to pesticides and specific crop uses that would be lost under FIFRA 1988 and EPA 1990. A committee of University of California pest management specialists and agricultural economists developed a survey asking respondents to provide information on the number and frequency of applications, application method, and target pest for each crop and targeted pesticide for which they felt they had sufficient expertise.

The questionnaire also asked for alternative pesticides, their method and frequency of application, expected yield and quality compared to those with the targeted pesticide, and deficiencies or problems with the proposed alternative. Similarly, it asked for nonchemical alternatives or alternative systems, the cost of the alternative or the data required to determine that cost, the yield and quality compared to those with the targeted pesticide, and deficiencies or constraints to the use of the alternative. The questionnaire and a draft of Stimmann and Ferguson's article Potential pesticide use cancellations in California went to 140 Cooperative Extension advisors and specialists, Agricultural Experiment Station faculty, and USDA-Agricultural Research Service researchers with experience in pest management on California agricultural crops. Seventy people responded with 760 completed questionnaires. A database was assembled that classified and summarized the responses by crop, target pesticide, and target pest.

In the next step, a specialist from each of the pest management disciplines - plant pathology, nematology, entomology, and weed science - was asked to assemble a workgroup of other research and extension experts in his or her discipline to review the database summaries and to reach a consensus on each survey item, including establishment of the present availability of each alternative (ignoring cost), correction of any inaccuracies, and filling in of data gaps. The four workgroups comprised more than 40 individuals. The results were added to the database.

Pest management methods were categorized as biological, cultural, or chemical, with further subdivisions as follows:

\section{Biological alternatives}

- Biological control by multicellular organisms - including release of exotic parasites and predators, conservation and augmentation of natural enemies, genetic improvement of natural enemies, and allelopathy

- Biological control by microbial agents - application of beneficial or antagonistic living microorganisms or toxins synthesized by microbial agents

- Management practices - including natural mulches, living mulches, trap crops, and cover crops to enhance natural enemies or to control pest species

\section{Organically acceptable chemical alternatives}

- Oils and soaps - some horticultural oils and various fatty acids

- Botanicals - toxins derived from plants, such as pyrethrum and ryania

- Semiochemicals - pheromones, allomones, and kairomones, including sex attractants, feeding attractants, and repellants produced by insects and affecting the behavior of other insects

- Inorganic or elemental compounds - such as elemental sulfur and some copper formulations

\section{Synthetic organic chemicals}

- Synthetic organic pesticides - including chlorinated hydrocarbons, organophosphates, carbamates, pyrethroids, and insect growth regulators

- Fertilizers - use of commercial fertilizers in control of a pest species

\section{Cultural alternatives}

- Crop rotation - rotation of various lengths and fallow periods

- Physical controls - such as tillage, mowing, chopping, and flaming

- Sanitation - removing noncrop hosts and infested hosts

- Pruning and canopy management - physically manipulating the structure of the host plant

- Irrigation management - controlling water application and drainage

- Strategic choices - choice of field, location, planting and intended harvest dates, vigorous cultivars, plant density, transplanting, etc.

- Regulation - including mandatory host-free periods, hostfree zones, crop termination, seed indexing, and detection

\section{Genetics and plant improvement}

- Host plant resistance - including cultivars and rootstocks

\section{Status of the database}

All the data that have been received have been entered into the database. The data address pesticides targeted by FIFRA 1988 and EPA 1990, including 14 fungicides, 12 herbicides, 26 insecticides, and 1 nematicide. Data for the fungicides are available on 44 crops, for the herbicides on 45 crops, for the insecticides on 60 crops, and for the nematicide on 63 crops. Almost 600 crop and pest situations are addressed. The database includes biological, cultural, or chemical alternatives for each targeted pesticide, crop, and pest identified by the specialists who contributed the information. The current status of a given alternative (i.e., its availability) is also provided. 
No further attempt has been made to quantify the actual impacts of potential alternatives on crop yield or quality, or to assess the economics of using an alternative control identified in the database. In subsequent analyses, economists will combine the information now in the database with costs for materials, labor, and other factors. The current data have been returned to UC pest management experts who will fill the data gaps and supply information on alternatives for use on any specific crops and pests not now addressed in the database.

\section{Preliminary review}

Table 1 summarizes a portion of the database for field crops, including beans, cotton, grain (barley, corn, oats, and wheat), hay (alfalfa), rice, and sugarbeets; fruit and nut crops, including almond, citrus, grape, pome fruits (apples and pears), soft fruits (apricots, nectarines, peaches, and plums), strawberry, and walnut; and vegetables, including carrot, celery, cole crops (broccoli, Brussels sprouts, cabbage, and cauliflower), lettuce (head and leaf), melon, onion, potato, and tomato. These crops and crop groups represent over $82 \%$ of California's $\$ 9$ billion crop production industry. For each crop or cropgroup and targeted pesticide, the table indicates the biological, cultural, or chemical alternatives listed in the database as currently available.

The notion of currently available must be considered. Although the table lists an alternative for a pesticide, the alternative may not be appropriate for all pests controlled by that targeted pesticide. Other alternatives that would require additional research or registrations before becoming available for use were also supplied by the respondents. These appear in the database, but are not included in table 1 . The database indicates that alternatives are available for many targeted pesticides and crops, though they are not necessarily acceptable because of costs or other constraints.

Of the available alternatives, $60 \%$ are chemicals, many of them synthetic organic pesticides. At least one biological or chemical alternative was noted for $75 \%$ of the specific crop-pest combinations where some alternative was identified. The fewest alternatives of any kind are available for pre- and post-harvest fungicides that would be lost as a result of the regulations. For example, no alternatives were identified for some crop uses of such important materials as benomyl. Few available nonchemical alternatives were identified for many crops and targeted fungicides.

\section{True availability of alternatives}

Although the database lists about $70 \%$ of the alternatives as available, the true practical and economic availability of many is uncertain. Some alternative pesticides, for example, are under review for water or air quality considerations, and in the near future they may be unavailable because of other regulatory actions. Some are not effective in all production areas because of environmental factors that limit their use or efficacy, or because of pesticide resistance. Some of the alternative pesticides are more disruptive to natural enemies or may result in more hazard to field workers than the pesticides in current use. Efficacies and application techniques for alternative chemicals are not always known.

Similarly, implementation of some nonchemical control alternatives reported as available might be considered impractical in some situations, either because they are not perceived as cost-efficient or because they conflict with current production practices.

Most weeds can be managed by physical means such as cultivation, hand-pulling, or hoeing. Solarization with plastic mulch is another alternative. In practice, these nonchemical methods are not widely used by growers, or are used sparingly and in combination with herbicides because of their economic and labor costs. Some crops compete better with weeds than others, and require more or less intensive weed management depending on the specific situation. Some mature orchard crops can probably tolerate a managed weed cover, but many field and vegetable crop yields are reduced in the presence of only a few competitors, and require more com- plete weed control. Current and proposed legislation restricts the number of herbicide options, and economic considerations will be important to producers and consumers as the move continues to new chemical and nonchemical alternatives.

Even where an alternative pesticide is identified, biological efficacy or environmental concerns may limit its practicality. For example, phosmet is a targeted insecticide for control of codling moth on pome fruit. The only available chemical control identified in the database is azinphosmethyl. The moth's resistance to azinphosmethyl was identified during 1989 in the Sacramento Delta, raising questions about the long-term utility of this chemical. Furthermore, azinphosmethyl is under study as a potential toxic air contaminant, and that could lead to regulatory action. Two microbial agents Bacillus thuringiensis and a granulosis virus - could be used for control, but the efficacy or cost of these agents could limit their widespread applicability. Using early maturing varieties in order to avoid exposing fruit to multiple pest generations and high lateseason populations, bagging individual fruit on the tree to exclude the pest, and trunk banding to capture and remove the insect were all considered to be available, nonchemical options. Planting early season varieties could be a limited option because replanting is costly, markets favor traditional varieties, and the market period would be severely restricted. Bagging individual fruit is a highly effective tactic, but its cost would be high. Similarly, trunk banding would be labor intensive, and its relative efficacy is not well known.

The database lists a chemical control (copper sulfate) and a cultural control (draining paddy water) as available alternatives to parathion for tadpole shrimp control in rice. Copper sulfate is more expensive than parathion. Furthermore, copper is a heavy metal with algacidal properties whose environmental impacts, if widely used, are not well known. Draining paddy water can release herbicides into drainage canals, a known concern when detected in the Sacramento River. Draining paddy water also promotes weed growth, and because that can lead to reduced yields it may prompt an additional herbicide application. In effect, the cultural alternative could promote application of a different category of chemical.

In recent years, nematologists have been forced to seek control alternatives more aggressively than researchers in other pest management disciplines because so many nematode control chemicals have been canceled. Possibly as a result of this need, the database for nematode control alternatives is very thorough. Use of the one nematicide considered in this study, 1,3-dichloropropene, has been suspended since we assembled the preliminary database. Perhaps its most broadly available chemical alternative, methyl bromide, is really of limited use except in high-value crops, since fields must be completely tarped with plastic before the material is injected. This type of treatment is impractical on a large scale, given current technology. In addition, worker safety would be of special concern with more widespread production and use of methyl bromide. Another repeatedly listed alternative was the practice of crop rotation. Traditionally, nematodes were often controlled in annual systems by the extensive use of crop rotation or fallow periods. For various reasons involving economics and land availability, these nonchemical techniques are no longer in extensive use. Whether they will again be seen as viable management alternatives will depend on alternate uses of land and equipment. Many traditional rotations may not be economically viable. However, recent advances in genetic resistance to nematodes for some crops (e.g.,tomatoes) make rotations that include nematode-resistant cultivars a possibility.

The chemicals shown in boldface in table 1 are the targeted pesticides for which our questionnaires showed no response and the six chemicals that were added to the Environmental Protection Agency's list of potential carcinogens after we had assembled the preliminary database. Until UC pest management specialists complete their further review of the database, we cannot tell whether these data gaps represent errors of omission or a true lack of alternative controls. 
TABLE 1. Availability of biological agents, chemicals (organically acceptable or synthetic), cultural practices, or host plant resistance as alternatives for current pesticide uses (where the table lists no alternatives, boldface indicates a gap in the preliminary database and plain type indicates that the database shows no alternatives)

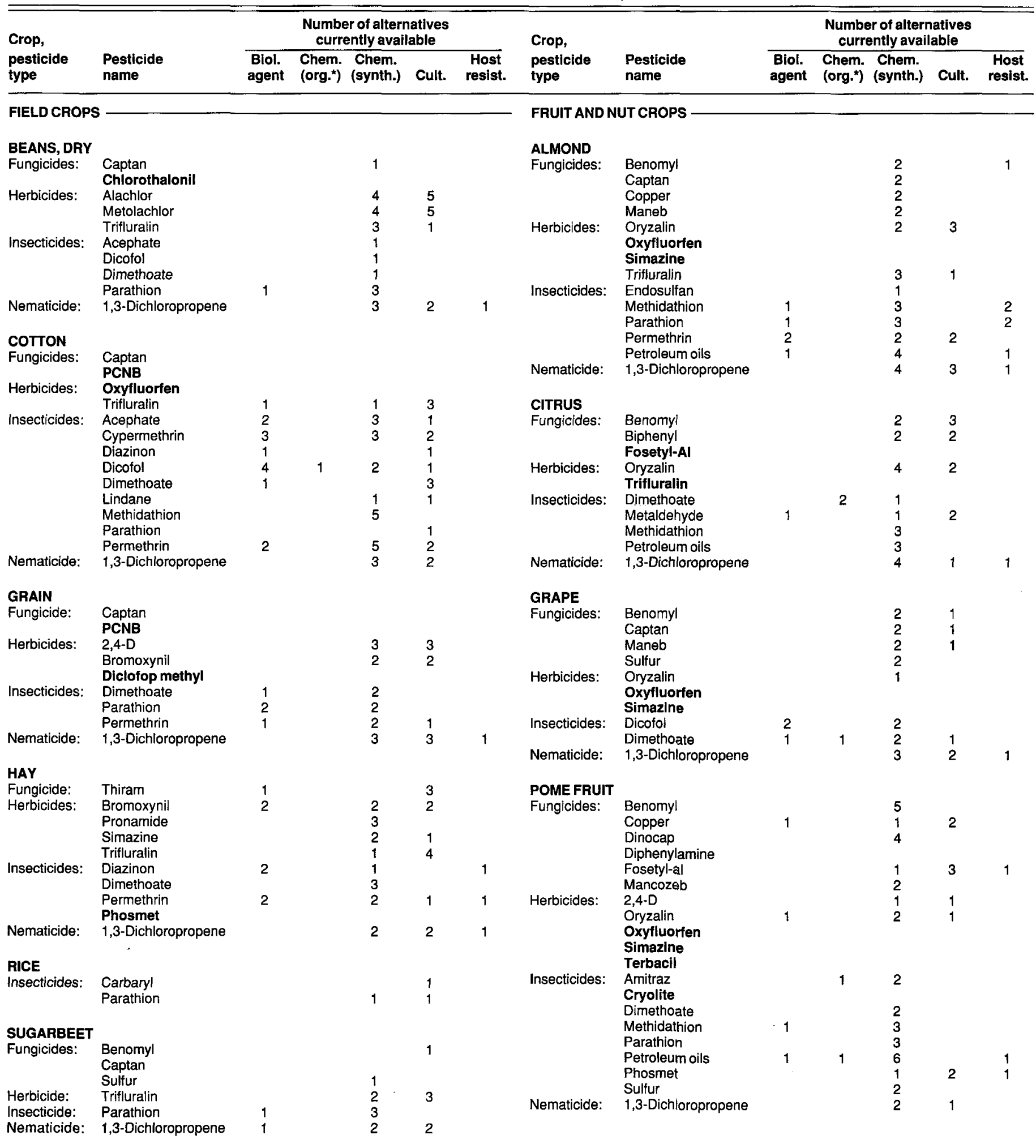

*Organically acceptable chemical alternatives, consistent with the California Health and Safety Code, Section 26569.11 , as enacted by the California Organic Food Act of 1979, amended 1982. 
TABLE 1. (continued) Availability of biological agents, chemicals (organically acceptable or synthetic), cultural practices, or host plant resistance as

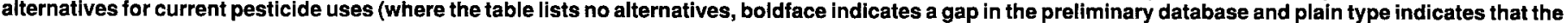
database shows no alternatives)

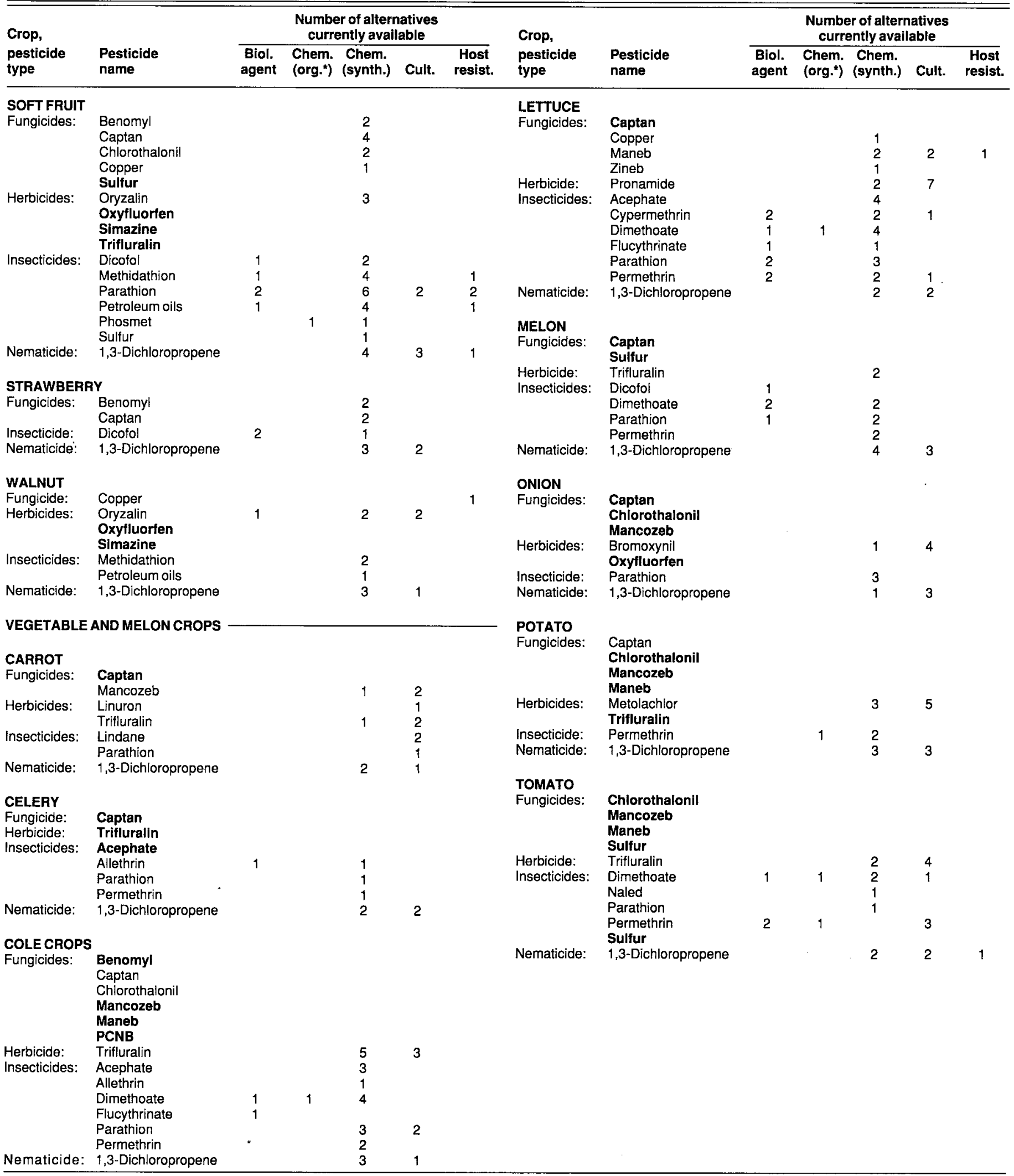

"Organically acceptable chemical alternatives, consistent with the California Health and Safety Code, Section 26569.11 , as enacted by the California Organic Food Act of 1979, amended 1982. 


\section{Beyond the current database}

The database of alternatives will need to be updated continually with changes that occur as the result of regulations and their interpretation. However, even in its current preliminary state, it serves several important uses. The database can now be used to identify crop and pest situations in critical need of research. It can also be used to generate preliminary assessments of the economic impact of specific control practices or of restrictions on their use.

Information in the database must be applied carefully, however, because the alternative control strategy that will ultimately be used and even its degree of universal availability may not be readily apparent. Cost and benefit considerations will affect the individual grower's choice of control strategy. We can assume that the grower will choose options that will maintain his or her competitive advantage with other growers. The analysis becomes more complex when social and environmental factors are included. Even when regulations restrict the options of all growers, and assuming that some of the restrictions would influence the cost, quality, or availability of some products, market forces will undoubtedly continue to influence what growers can produce and at what production level. Consumers of raw agricultural products, including packers and processors as well as the general public, will help to clarify decisions for growers based upon their reaction to the changes they perceive in the cost or quality of products. This might result in changes to crop production systems that we cannot predict accurately at this time.

Frank G. Zalom is Director, Statewide IPM Project, IPM Implementation Group, and Extension Entomologist, based at UC Davis; and Joyce F. Strand is Computer Systems Manager, IPM Implementation Group, also at UC Davis.

The pesticide alternatives database discussed in this paper will be available in early August, 1990 . For ordering information, write or call: Statewide IPM Project, IPM/IG, University of California, Davis, California 95616, telephone (916) 752-8350.

\title{
The research imperatives: knowledge to reduce the use of broadly toxic pesticides
}

\author{
Mary Louise Flint
}

The University of California has been a leader in the development of methods to reduce reliance on pesticides. California was the site of the first major successes of biological control for insect pests during the latter part of the 19th century, and the term integrated control (the forerunner of integrated pest management) was coined by UC entomologists in the late 1950s.

California has continued as a leader in innovative pest management, making pioneering contributions in the areas of genetic improvement of crop plants and natural enemies, microbial control, cover crops, cultural controls, use of pheromones, and selective use of pesticides within integrated pest management (IPM) programs. As Zalom and Strand show in the preceding paper, this research has resulted in many successful applications. However, much more progress would be required to eliminate all need for the pesticides targeted by the California Environmental Protection Act of 1990 (EPA 1990) and the ongoing FIFRA reregistration process.

Most pesticide alternatives are very specific in their action, so they must be developed individually for each pest and crop situation. For instance, most biological control agents are effective only against one pest or a small group of pests. The narrow range of affected organisms is part of what makes these techniques more environmentally sound than broad-spectrum pesticides, but it also accounts for their limited development. The grower normally has a dozen or more major pests to contend with in any given crop; providing an alternative for just one pest won't be very helpful unless it is part of an IPM program that considers appropriate, nondisruptive ways to manage the other pests as well.

This points to the need to develop ecologically based, crop-oriented IPM programs that will become part of the overall crop or animal production system. Few of these techniques will be adopted unless research and extension workers can adapt and disseminate them in appropriate ways and demonstrate their economic viability.

The discussion below outlines the biological, cultural, chemical, and issue-related areas of research that are likely to lead to a reduction in the use of broad-spectrum pesticides. After a brief description of each area, its critical research needs are listed. Substantial expertise in many of these areas already exists at the University of California and elsewhere, and much research is in progress. How- ever, these efforts will have to accelerate in order to bring techniques rapidly to the field and broaden their application.

\section{Biological options}

- Classical biological control involves the deliberate introduction and establishment of natural enemies where they have not previously occurred. Most classical biological control programs target pests of exotic origin that have accidentally been introduced into new areas, and become established in the absence of their key natural enemies. Often, introduction of a well-adapted natural enemy can bring the pest under such complete control that it requires no further pest management action. This technique has proven successful against many insects and weeds.

Critical research needs: better understanding of the ecology of target pests and their natural enemies under the full range of climatic conditions in California; more foreign exploration; better facilities for rearing imported beneficials; better techniques for evaluating success

- Augmentative release of natural enemies involves the mass rearing of natural enemies in a rearing facility, and their subsequent release in pest-infested fields. Unlike classical biological control, this technique generally requires the periodic release of large numbers of natural enemies for adequate pest management. Predaceous or parasitic insects, mites, and nematodes have been used in this manner. Microbial agents are covered below.

Critical research needs: better rearing techniques; development of artificial host media; better release techniques; knowledge of timing of releases

- Cover crops and living mulches are resident or planted noncrop species grown on an orchard floor or on fallow land to reduce undesirable pest populations or provide other benefits. When used as mulches (either living or killed), they allow annual crops to be planted with nontillage or reduced tillage. Weeds may be suppressed through physical competition or allelopathy. Nematodes may be suppressed through antagonistic or allelopathic effects, or by preferential attraction to the cover crop (also called trap crop) over the desired crop. Cover crops also may provide habitat for natural enemies of insects, mites, and other pests.

Critical research needs: more knowledge about the impact of cover 